Document downloaded from:

http://hdl.handle.net/10251/92407

This paper must be cited as:

Chicharro López, FI.; Ortega Tamarit, B.; Mora Almerich, J. (2016). Paired SSB optical OFDM channels for high spectral efficient signal transmission over DWDM networks. Optics Communications. 370:239-244. doi:10.1016/j.optcom.2016.03.007

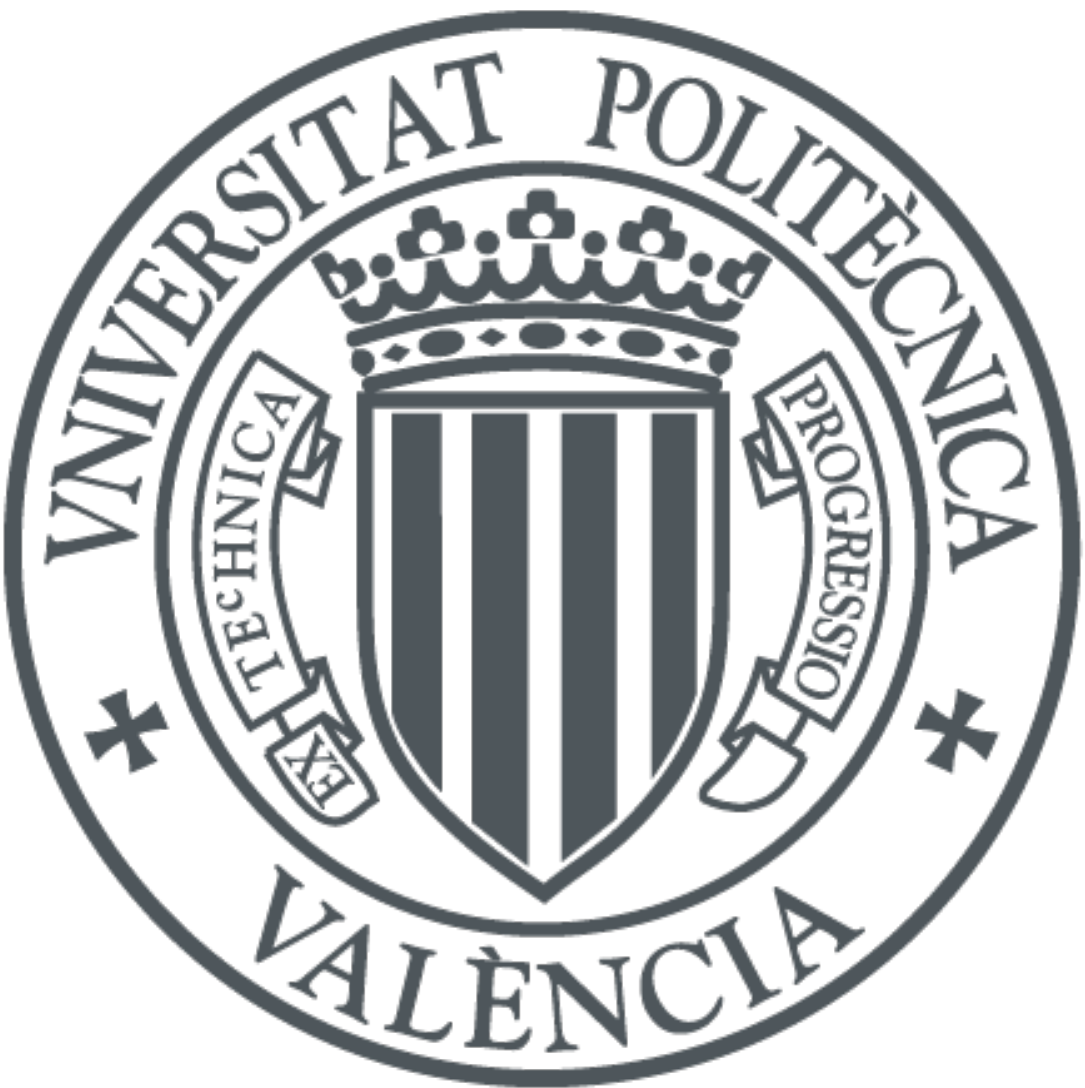

The final publication is available at

http://doi.org/10.1016/j.optcom.2016.03.007

Copyright Elsevier

Additional Information 


\title{
Paired SSB Optical OFDM Channels for High Spectral Efficient Signal Transmission over DWDM Networks
}

\author{
Francisco Chicharro, Beatriz Ortega, Member, IEEE, José Mora ${ }^{1}$ \\ ITEAM Research Institute, Universitat Politècnica de València, Camino de Vera $s / n$, \\ 46022 Valencia, Spain
}

\begin{abstract}
A new high spectral efficient SSB-OOFDM DWDM transmission system has been experimentally demonstrated. The proposed transmitter employs paired optical channels consisting of two SSB modulated OFDM signals using opposite sidebands in order to allow an efficient use of the spectrum with optical carriers separation under $10 \mathrm{GHz}$. Moreover, different paired channels are multiplexed into the $25 \mathrm{GHz}$ grid DWDM fiber transmission link. Optical carrier spacing of $8.75 \mathrm{GHz}$ in paired channels has been demonstrated allowing $40.8 \mathrm{~Gb} / \mathrm{s}$ signal transmission rate over a $25 \mathrm{GHz}$ paired channel bandwidth.
\end{abstract}

Keywords: Optical networks, OFDM, dense wavelength, division multiplexing, direct detection.

\section{Introduction}

The increasing demand of bandwidth in optical telecommunication networks for latest videogames, cloud computing or videoconference services, amongst others, leads them to employ advanced modulation formats exploiting the recent 5 digital signal processing techniques. Orthogonal Frequency Division Multiplexing (OFDM) is a well-known modulation technique employed in various standards, such as DVB, LTE, WiFi or WiMAX. The use of OFDM in optical networks has attracted lot of interest during the last years due to its high spectral

\footnotetext{
${ }^{1}$ E-mail address: jmalmer@iteam.upv.es
} 
efficiency, flexibility and robustness to overcome the fiber chromatic dispersion effect [1, 2].

Optical OFDM signal can be transmitted by using either coherent optical OFDM (CO-OFDM) or incoherent intensity modulated and directly detected (IM-DD) OOFDM. The former shows better spectral efficiency and lower optical signal to noise ratio (OSNR) sensitivity although its phase noise impairment requires expensive and complex equipment, which makes it suitable for long haul transmission. However, the latter shows relaxed laser linewidth requirements and receiver simplicity, and leads to a promising approach for cost sensitive scenarios such as Metropolitan and Local Area Networks (MANs/LANs) 3 .

Low cost and compact IM-DD OOFDM transmitters based on directly modulated lasers provide extintion ratio and frequency chirp strongly dependent on the DML operating conditions. Carrier suppression is usually required in these approaches because of the large power ratio between the optical carrier and the optical OFDM band. The alternative employs external modulation using electro-optic Mach-Zehnder modulators (MZM), which will also reduce the modulation nonlinearities. In both modulation schemes, conventional double sideband (DSB) modulated signals lead to chromatic dispersion induced power fading which is avoided by using optical single sideband (SSB) 1]. The SSB signal generation requires narrow optical filtering or special modulators [4] with increased costs and complexity. However, successful experimental results

30 in the literature over $80 \mathrm{~km}$ and $30 \mathrm{~Gb} / \mathrm{s}[3$ ] confirm this modulation format as a preferred option for OOFDM signal transmission. Furthermore, in the literature, different approaches have been previously proposed towards increasing the transmission capacity of such systems. One example is the modulation of two independent OFDM bands onto the opposite side on the optical carrier in [5] 35 by using a dual-electrode MZM. In this approach, the required power clipping due to the high modulation indexes implies a significant BER penalty. Besides the impact of nonlinear mixing must be minimized while maximum optical bandwidth efficiency is kept.

Another approach is based on compatible SSB modulation, which consists 
of modulating the envelope of the optical power instead of the amplitude of the field, as proposed in [6, 7]. The required bandwidth of these systems has been drastically reduced since this proposal does not require the spectral gap between the carrier and the OFDM band. However, the increased carrier to signal power ratio and the use of a complex IQ modulator are its main drawbacks,

45 partially overcome by the proposal of using a single dual electrode MZM [8]. This modulation provides double bandwidth efficiency compared to IM-OFDM with increased OSNR, but nonlinearities still need to be improved.

Iterative detection techniques by using digital signal processing algorithms have been also proposed to cancel signal to signal beat interference $[9$. Therefore, guard band can be reduced in order to demonstrate high spectral efficient and flexible optical metro networks [10].

In this paper, we propose and experimentally demonstrate a spectrally efficient technique to transmit paired SSB optical OFDM channels over DWDM networks. Each set of paired channels employs two optical carriers, which are SSB modulated with opposite sidebands. Both channels are multiplexed in an optimized schematic which allows to allocate them closer in the spectrum to demonstrate enhanced aggregated bit rates by using moderate costs equipment. The paper is structured as follows: Section 2 describes the paired DWDM-SSBOOFDM transmitter, Section 3 presents the experimental results and finally, 60 Section 4 summarizes the main conclusions of the paper.

\section{Description of the Paired DWDM-SSB-OOFDM Transmitter}

Figure 1 shows the paired DWDM-SSB-OOFDM channels transmitter where channels \#i are composed of two adjacent optical carriers which identify the channels \#i1 and \#i2. Taking profit from the SSB modulation, both optical carriers are closely located in the spectrum by means of suppressing modulation bands between them. In a DWDM network using $\mathrm{N}$ channels, the transmitter will require $2 \mathrm{~N}$ DFB lasers generating different optical carriers, which are double sideband modulated by the OFDM signals using external electro-optic MZMs. 


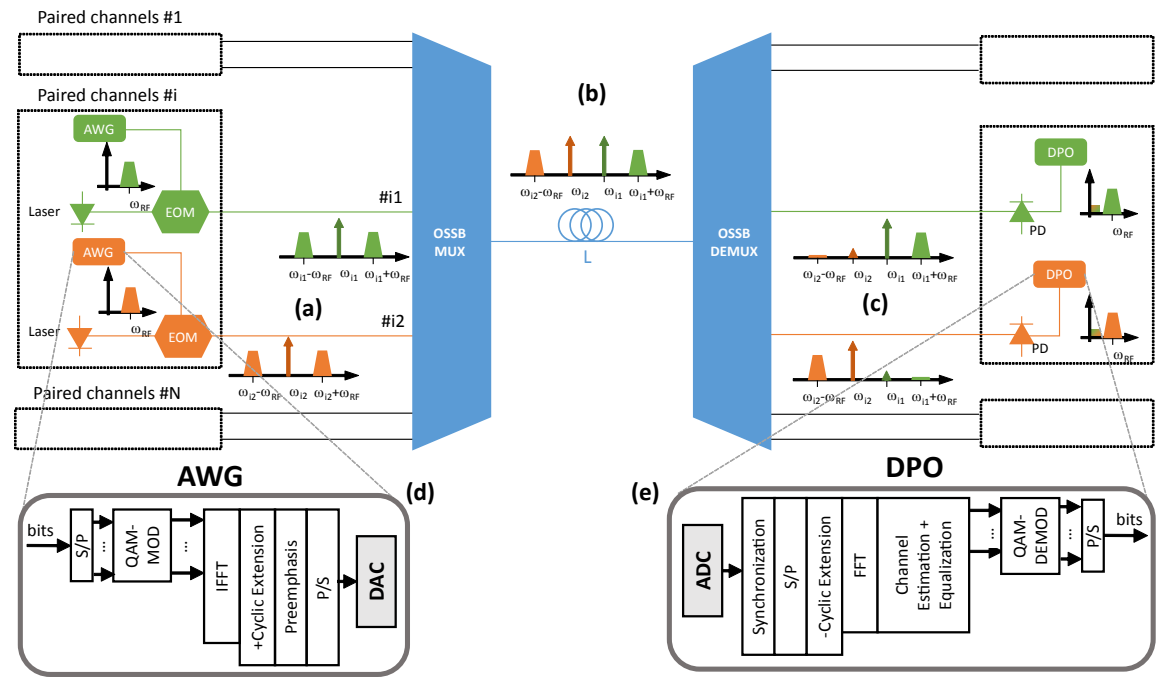

Figure 1: Layout of the paired DWDM-SSB-OOFDM channel transmitter. Insets: (a) DSB modulated signal using both optical carriers composing paired channels $(\mathrm{CH} \# \mathrm{i})$ ( $\mathrm{CHi}$ and CHi2), (b) Paired (multiplexed) SSB-OOFDM channels before transmission through fiber link and (c) Demultiplexed SSB modulated signals, (d) Digital OFDM transmitter, (e) Digital OFDM receiver.

A $20.4 \mathrm{~Gb} / \mathrm{s}$ data stream was transmitted by using the scheme depicted in inset (d) of Fig. 1. A parallelized bit stream was 16QAM modulated by using 480 subcarriers of an OFDM signal. Hermitian symmetry must be satisfied in the 2048-point IFFT for a real signal and a cyclic prefix of $1 / 32$ of the symbol is appended. Finally, the generated data is serialized and loaded into an 24 GS/s Arbitrary Waveform Generator (AWG7122C, Tektronix). The complete OFDM frame has a duration of $10.733 \mu$ s and it is composed by one preamble symbol for synchronization, 114 data symbols and 7 pilot symbols, which are distributed amongst the data symbols for channel equalization. The electrical signal is centered at $2.9 \mathrm{GHz}$ and it ranges up to $5.875 \mathrm{GHz}$, using reduced guard band at the expense of optimized carrier to sideband ratio of the optical so signal [11].

Each DWDM channel requires an optical channel bandpass multiplexer with two input ports which eliminates one of the bands of each optical DSB modula- 

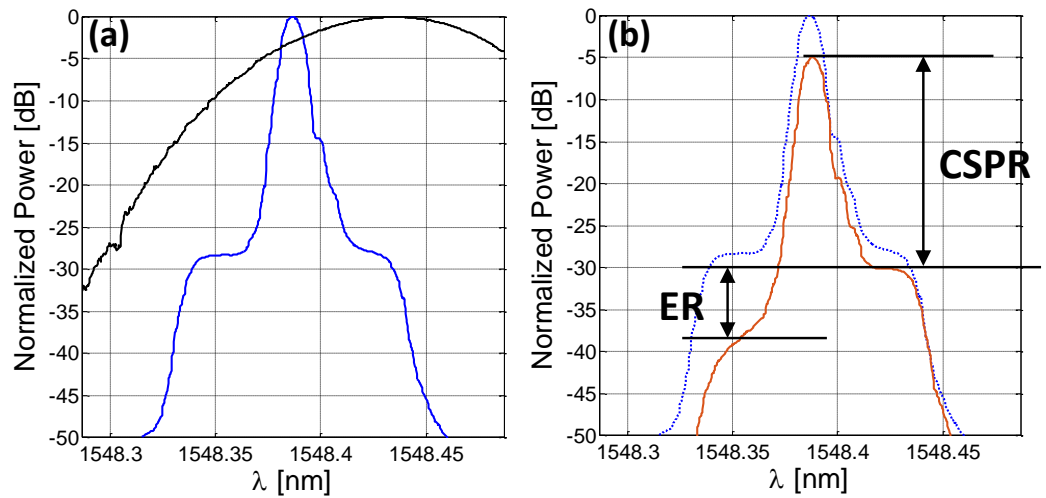

Figure 2: (a) Normalized optical spectrum of the DSB modulated signal (blue) and filter transmission bandpass (black), (b) DSB (dotted blue line) and SSB (solid orange line) modulated signal.

ted signal and also multiplexes them into the same fiber to be transmitted over the network. Thus, a $2 \mathrm{~N}$ input ports bandpass multiplexer will be required by a $\mathrm{N}$ paired channels DWDM transmitter, as depicted in Fig. 1. and the corresponding bandpass demultiplexer will be necessary prior to photodetectors (PD) in order to extract every optical OFDM transmitted signal. As an example, Fig. 2 (a) shows the optical spectrum of the DSB modulated optical channel \#i2 set at $1548.38 \mathrm{~nm}$ and the optical filter transmission transfer function, measured with a $0.01 \mathrm{~nm}$ spectral resolution. The uneven sidebands are due to the spectral asymmetry of the laser output signal. The optical filter consists of a multiport tunable optical filter (WaveShaper 4000S, Finisar). This filter permits to tune the central frequency from 1527.4 to $1567.5 \mathrm{~nm}$ with steps of $77 \mathrm{pm}$; the $3 \mathrm{~dB}$ bandwidth is set at the minimum value of $0.1 \mathrm{~nm}$ and the corresponding fixed roll-off is $0.06 \mathrm{~dB} / \mathrm{pm}$. The output signal stability after filtering stage is similar to the EXFO DFB laser stability, which exhibits $0.03 \mathrm{~dB}$ power stability and $0.002 \mathrm{~nm}$ wavelength stability at room temperature along $8 \mathrm{~h}$, and therefore, stable system performance is guaranteed. Consequently, Fig. 2(b) plots the resulting SSB modulated signal with the carrier and upper sideband transmitted after the first OSSB filter and the previous DSB modulated signal. An insertion 
loss around $5 \mathrm{~dB}$ is measured in the optical filter. Optical wavelength of channel \#i1 was set at $1548.29 \mathrm{~nm}$, and was SSB modulated but using the transmitted lower wavelength sideband.

The quality of the SSB modulation can be characterized in terms of the Carrier to Sideband Power Ratio (CSPR) and the Extinction Ratio (ER) [3]:

$$
C S P R=\frac{P_{C}}{P_{U S-S S B}}, \quad E R=\frac{P_{U S-S S B}}{P_{L S-S S B}},
$$

where $P_{C}, P_{U S-S S B}$ and $P_{L S-S S B}$ are the carrier, upper and lower sideband optical powers using SSB modulation, respectively. After the signal was transmitted through $50 \mathrm{~km}$ SMF link, a second tunable filter was used to select each optical OFDM channel before signal detection. Finally, after detection of each demultiplexed channel by a photodiode, the received electrical signal is sampled at $50 \mathrm{GS} / \mathrm{s}$ by a real time digital oscilloscope (DPO72004C, Tektronix). The digital offline postprocessing blocks are shown in inset (e) of Fig. 1. After the synchronization stage, the data is parallelized. The cyclic prefix is removed and the FFT is applied. The block-type pilots are used for channel estimation and signal equalization. Data is demodulated and the transmitted bit stream is recovered after the serialization stage.

\section{Experimental Results}

The quality of the SSB signal depends on the optical filter transfer function, the relative spectral position of the central wavelength of the optical bandpass filter, $\lambda_{F}$, and the optical carrier, $\lambda_{0}$. With $0 \mathrm{dBm}$ received optical power, both optical modulated signals have been characterized in terms of CSPR and ER for different detuning factor $\left(\lambda_{F}-\lambda_{0}\right)$, according to Eq. 1 as depicted in Fig. 3(a). As long as some displacement between the optical carrier and the center of the filter bandpass is introduced, the power of one of the modulated bands is being reduced respect to the other one. SSB modulation is achieved when high CSPR and ER values are obtained while signal degradation keeps low (see measured BER values in Fig. 3(b)). In our experimental setup, $0.045 \mathrm{~nm}$ 


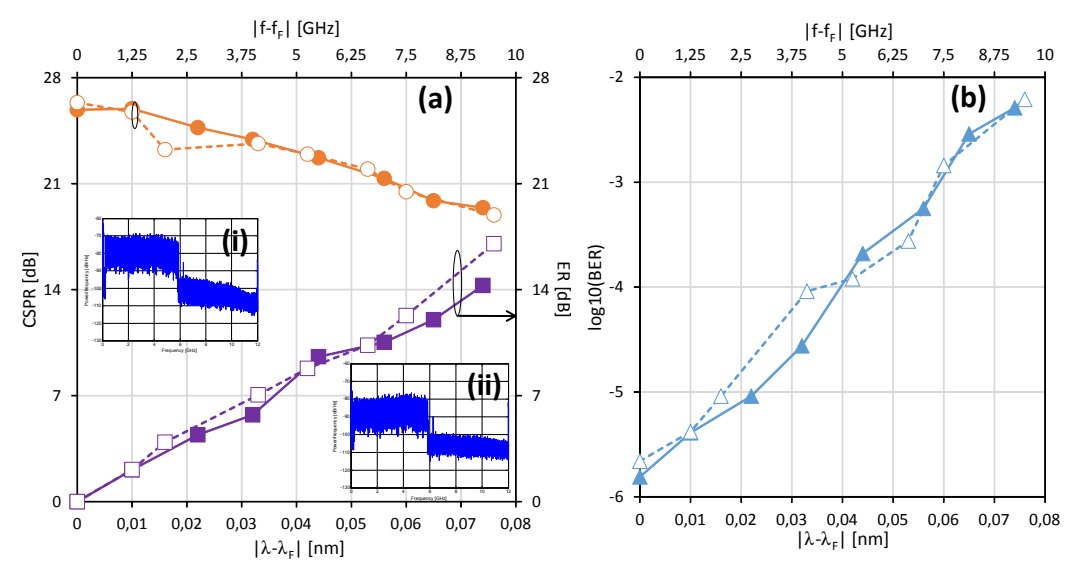

Figure 3: Quality of the SSB modulated signal: (a) CSPR (orange circles), ER (purple squares) and (b) BER (blue triangles) of the recovered data vs the spectral position of the optical filter bandpass respect to the optical carrier for both paired channels: \#i1 (filled) and \#i2 (blank). Insets: Electrical spectra for detuning factor equal to (i) $0.01 \mathrm{~nm}$ and (ii) $0.06 \mathrm{~nm}$

wavelength displacement was set in order to obtain a CSPR of $23 \mathrm{~dB}$ whereas ER is $10 \mathrm{~dB}$ and BER keeps lower than $10^{-4}$. The electrical spectra obtained with detuning factor of $0.01 \mathrm{~nm}$ and $0.06 \mathrm{~nm}$ are shown in Insets (i) and (ii) of Fig. 3, respectively.

Once both channels are correctly SSB modulated and multiplexed by the proposed system depicted in Fig. 1 the performance of paired multiplexed channels and its dependence on the channels spacing must be measured as follows. Since we aim to demonstrate the highest efficient spectral use, optical carriers of the paired channels are closely located with only 105 pm separation. Figure 4(a) shows the optical spectrum of paired SSB-signal before the link. Figure 4(b) depicts both demultiplexed channels after the $50 \mathrm{~km}$ fiber link showing a channel crosstalk around $15 \mathrm{~dB}$ and $18 \mathrm{~dB}$ for \#i1 and \#i2, respectively. The corresponding constellation diagrams are plotted in Fig. 4(c) and 4(d) for demultiplexed channels \#i1 and \#i2, respectively. The recovered signal quality is $\mathrm{BER}=1.55 \cdot 10^{-4}$ for channel $\# \mathrm{i} 1$ and $\mathrm{BER}=1.20 \cdot 10^{-4}$ for channel $\# \mathrm{i} 2$, corresponding to EVM values of $13.49 \%$ and $13.19 \%$, respectively, having -11.5 $\mathrm{dBm}$ as received optical power. 

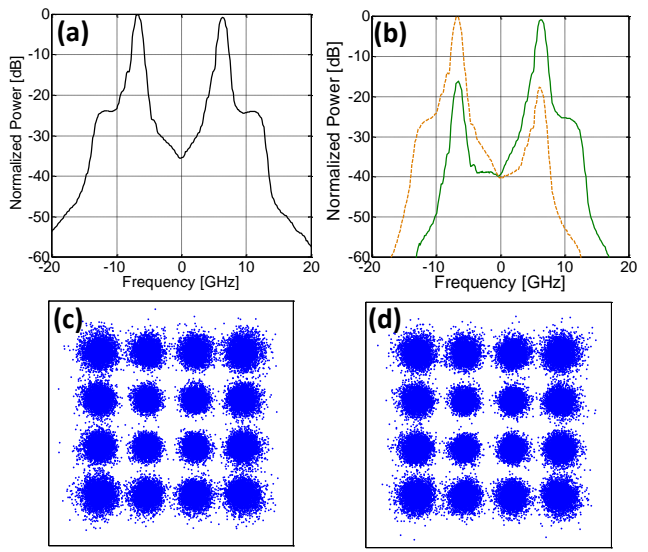

(d)

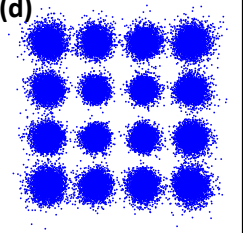

Figure 4: Optical spectrum of paired SSB-signal (optical carriers separation of $105 \mathrm{pm}$ ) before the link (a) and after the $50 \mathrm{~km}$ link (b) for demultiplexed channels \#i1 (solid green line) and \#i2 (dotted orange line). Constellation diagrams of the recovered data for channels \#i1 (c) and \#i2 (d).

Figure 5 shows the BER values of transmitted signals over $50 \mathrm{~km}$ for different channels spacing in the $[70-115]$ pm range. Insets (a) and (b) in Fig. 5 show the spectrum of both multiplexed OOFDM paired channels with 70 and $115 \mathrm{pm}$ spacing between them, respectively. Although an expected signal degradation is measured for closer optical carriers, BER values keep below $10^{-3}$ for such spacing interval. The crosstalk is measured as the ratio between the optical power of the selected and the unwanted transmitted channels, and reaches a minimum value of $6.8 \mathrm{~dB}$ when an optical power of $-11.8 \mathrm{dBm}$ is received. Therefore, a total bitrate of $40.8 \mathrm{~Gb} / \mathrm{s}$ has been successfully transmitted by a $25 \mathrm{GHz}$ channel bandwidth transporting two optical carriers spaced by $8.75 \mathrm{GHz}$ and modulated by 16QAM-uncorrelated OFDM signals. However, crosstalk and therefore the signal degradation can be further enhanced in order to allow higher total bit rates and higher signal quality, provided the OFDM signal generator supports it and the OSSB multiplexer satisfies the spectral requirements.

Finally, the paired SSB-optical OFDM transmitter has been tested in a DWDM network to evaluate the degradation of such channels. Three paired channels of $25 \mathrm{GHz}$ bandwidth have been transmitted over the network, each 


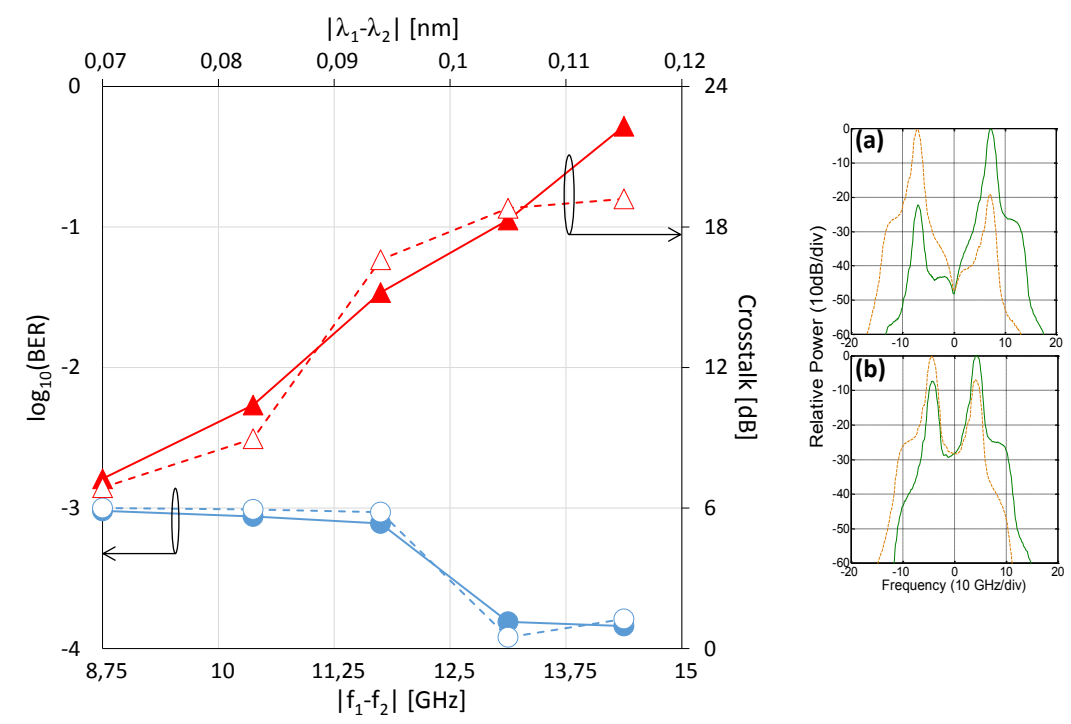

Figure 5: $B E R$ (blue circles) and crosstalk (red triangles) dependence on the wavelength spacing between multiplexed optical channels \#i1 (filled) and \#i2 (blank). Insets: optical spectrum when optical carriers are separated (a) $115 \mathrm{pm}$ and (b) $70 \mathrm{pm}$.

one transmitting $40.8 \mathrm{~Gb} / \mathrm{s}$, and degradation of both \#i1 and \#i2 of central channel has been evaluated. The spectra of the composed signal and also each demultiplexed \#i1 and \#i2 signals are shown in Fig. 6(a) and 6(b) with the corresponding measured constellations and obtained BER values of $5.6 \cdot 10^{-4}$ and $8.51 \cdot 10^{-4}$, respectively.

Experimental measurements of paired optical SSB modulated channels in a DWDM network demonstrate the viability of this proposal for increasing the capacity of such networks, although the use of properly designed SSB multiplexers is necessary to improve the extinction ratio of SSB generated signals as well as to increase the crosstalk values just prior to optical detection. Optimized filters will allow to increase the quality of the transmitted signal in real network scenarios. 

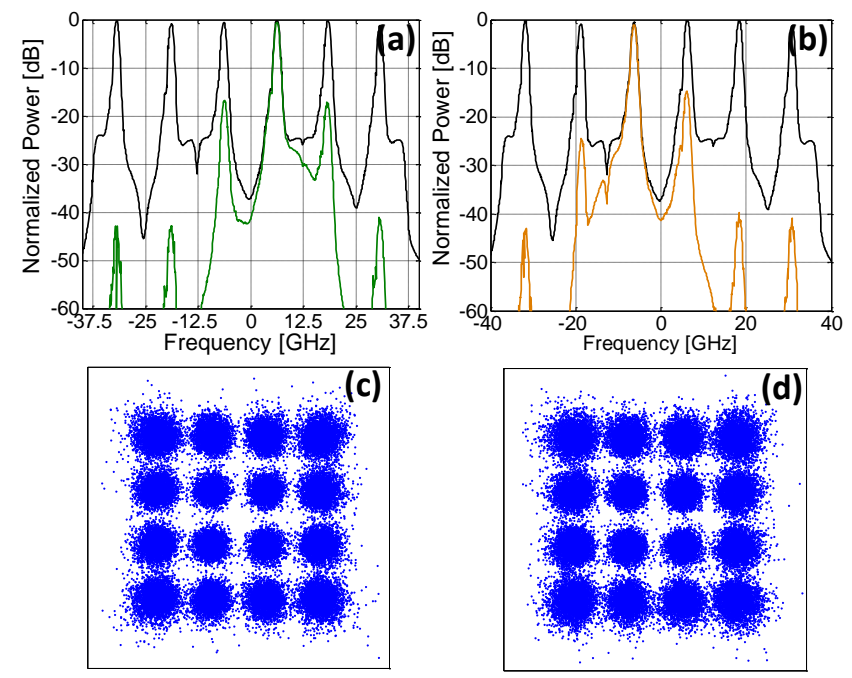

Figure 6: DWDM spectra of the transmitted signal (black line) and the demultiplexed spectra (\#i1 (green line) in (a), \#i2 (orange line) in (b)) and constellations of \#i1 (c) and \#i2 (d) demultiplexed signals.

\section{Conclusions}

A new paired SSB optical OFDM channels transmission system has been demonstrated with high potential for the future of optical OFDM networks. The system employs paired optical channels which are SSB modulated by optical filtering of the opposite sidebands. Both optical carriers can be modulated and closely spectrally located to demonstrate highest spectral efficiency and successful OFDM signal transmission. In this paper, we have experimentally demonstrated the successful transmission over $50 \mathrm{~km}$ fiber link with a total rate of $40.8 \mathrm{~Gb} / \mathrm{s}$ over each $25 \mathrm{GHz}$ paired channel bandwidth. Signal transmission over a DWDM network was demonstrated by using three different channels spaced by $25 \mathrm{GHz}$ and high capacity was demonstrated while $10 \mathrm{GHz}$ bandwidth moderate costs equipment was employed and scalability and flexibility of such networks were maintained. 


\section{Acknowledgements}

The research leading to these results has received funding from the national project TEC2011-26642 (NEWTON) funded by the Ministerio de Ciencia y Tecnología and the Research Excellency Award Programme GVA PROMETEO 2013/012 NEXT GENERATION MICROWAVE PHOTONIC TECHNOLOGIES.

\section{References}

[1] A. J. Lowery, J. Armstrong, Orthogonal-frequency-division multiplexing for optical dispersion compensation, Optics Express 14 (2006) 2079-2084.

[2] W. Shieh, C. Athaudage, Coherent optical orthogonal frequency division multiplexing, Electronic Letters 42 (2006) 587-589.

[3] J. Wei, X. Jin, J. Tang, The influence of directly modulated DFB lasers on the transmission performance of carrier-suppressed single-sideband optical OFDM signals over IMDD SMF systems, Journal of Lightwave Technology 27 (2009) 2412-2419.

[4] B. Lin, J. Li, H. Yang, Y. Wan, Y. He, Z. Chen, Comparison of DSB and SSB transmission for OFDM-PON [Invited], Journal of Optical Communications and Networking 4 (2012) B94-B100.

[5] Z. Xu, R. Hui, M. O'Sullivan, Dual-band OOFDM system based on tandem single-sideband modulation transmitter, Optics Express 17 (2009) 1347913486 .

[6] M. Schuster, S. Randel, C. A. Bunge, S. C. J. Lee, F. Breyer, B. Spinnler, K. Petermann, Spectrally efficient compatible single-sideband modulation for OFDM transmission with direct detection, IEEE Photonics Technology Letters 20 (2008) 670-672. 
[7] V. Vujicic, P. Anandarajah, C. Browning, L. Barry, WDM-OFDM-PON based on compatible SSB technique using a mode locked comb source, IEEE Photonics Technology Letters 25 (2013) 2058-2061.

[8] Z. Xu, M. O'Sullivan, R. Hui, OFDM system implementation using compatible SSB modulation with a dual-electrode MZM., in: Optical Fiber Communications Conference, San Diego, 2010, p. OMR2.

[9] W. Peng, B. Zhang, K. Feng, X. Wu, A. Willner, S. Chi, Spectrally efficient direct-detected OFDM transmission incorporating a tunable frequency gap and an iterative detection techniques, Journal of Lightwave Technology 27 (2009) 5723-5735.

[10] T. M. F. Alves, L. M. M. Mendes, A. V. T. Cartaxo, High granularity multiband OFDM virtual carrier-assisted direct-detection metro networks, Journal of Lightwave Technology 33 (2015) 42-54.

225 [11] J. Ma, W. Zhou, Joint influence of the optical carrier-to-sideband ratio and guard band on direct-detection SSB-OOFDM system, IEEE Photonics Journal 7 (2015) 7801713. 